\title{
Lansoprazole for secondary prevention of gastric or duodenal ulcers associated with long-term low-dose aspirin therapy: results of a prospective, multicenter, double-blind, randomized, double-dummy, active-controlled trial
}

\author{
Kentaro Sugano $\cdot$ Yasushi Matsumoto $\cdot$ Tsukasa Itabashi $\cdot$ Sumihisa Abe $\cdot$ Nobuhiro Sakaki \\ Kiyoshi Ashida $\cdot$ Yuji Mizokami $\cdot$ Tsutomu Chiba $\cdot$ Shigeyuki Matsui $\cdot$ Tatsuya Kanto \\ Kazuyuki Shimada $\cdot$ Shinichiro Uchiyama $\cdot$ Naomi Uemura $\cdot$ Naoki Hiramatsu
}

Received: 16 December 2010/ Accepted: 28 February 2011/Published online: 16 April 2011

(C) The Author(s) 2011. This article is published with open access at Springerlink.com

\begin{abstract}
Background The efficacy of low-dose lansoprazole has not been established for the prevention of recurrent gastric or duodenal ulcers in those receiving long-term low-dose aspirin (LDA) for cardiovascular and cerebrovascular protection. This study sought to examine the efficacy of low-dose lansoprazole (15 mg once daily) for the secondary prevention of LDA-associated gastric or duodenal ulcers.
\end{abstract}

For the Lansoprazole Ulcer Prevention Study Group (Low-Dose Aspirin Therapy).

Electronic supplementary material The online version of this article (doi:10.1007/s00535-011-0397-7) contains supplementary material, which is available to authorized users.

\section{K. Sugano $(\bowtie)$}

Division of Gastroenterology, Department of Internal Medicine, Jichi Medical University, 3311-1 Yakushiji, Shimotsuke,

Tochigi 329-0498, Japan

e-mail: sugano@jichi.ac.jp

\section{Y. Matsumoto}

Department of Neuroendovascular Therapy,

Kohnan Hospital, Nagamachi-Minami,

Taihaku-ku, Sendai, Miyagi 982-8523, Japan

T. Itabashi

Hokusetsu General Hospital, 6-24 Kita-Yanagawacho,

Takatsuki, Osaka 569-0000, Japan

S. Abe

Koukan Clinic, 1-2-1 Koukan Dori, Kawasaki-ku,

Kawasaki, Kanagawa 210-0852, Japan

\section{N. Sakaki}

Ebara Hospital, Tokyo Metropolitan Health and Medical

Treatment Corporation, 4-5-10 Higashi-Yukigaya,

Ota-ku, Tokyo 145-0065, Japan
Methods Patients were randomized to receive lansoprazole $15 \mathrm{mg}$ daily $(n=226)$ or gefarnate $50 \mathrm{mg}$ twice daily $(n=235)$ for 12 months or longer in a prospective, multicenter, double-blind, randomized active-controlled trial, followed by a 6-month follow-up study with open-label lansoprazole treatment. The study utilized 94 sites in Japan and 461 Japanese patients with a history of gastric or duodenal ulcers who required long-term LDA therapy for cardiovascular and cerebrovascular disease.

Results The primary endpoint was the development of gastric or duodenal ulcers. The cumulative incidence of gastric or duodenal ulcers on days 91, 181, and 361 from the start of the study was calculated by the Kaplan-Meier method as $1.5,2.1$, and $3.7 \%$, respectively, in the lansoprazole group versus $15.2,24.0$, and $31.7 \%$, respectively, in the gefarnate group. The risk of ulcer development was

\section{K. Ashida}

Department of Gastroenterology, Osaka Saiseikai Nakatsu

Hospital, 2-10-39 Shibata, Kita-ku, Osaka 530-0012, Japan

Y. Mizokami

Department of Gastroenterology, Tsukuba University Hospital,

2-1-1 Amakubo, Tsukuba, Ibaraki 305-8567, Japan

\section{T. Chiba}

Department of Gastroenterology and Hepatology,

Graduate School of Medicine, Kyoto University,

Yoshida-Konoecho, Sakyo-ku, Kyoto 606-8501, Japan

S. Matsui

Department of Data Science, The Institute of Statistical

Mathematics, 10-3 Midorimachi, Tachikawa,

Tokyo 190-8562, Japan

T. Kanto $\cdot$ N. Hiramatsu

Department of Gastroenterology and Hepatology,

Osaka University Graduate School of Medicine,

Yamadaoka, Suita, Osaka 565-0871, Japan 
significantly (log-rank test, $P<0.001$ ) lower in the lansoprazole group than in the gefarnate group, with the hazard ratio being 0.099 (95\% confidence interval [CI] 0.042-0.230).

Conclusion Lansoprazole was superior to gefarnate in reducing the risk of gastric or duodenal ulcer recurrence in patients with a definite history of gastric or duodenal ulcers who required long-term LDA therapy.

Keywords Low-dose aspirin - Gastric or duodenal ulcers - Lansoprazole - Cardiovascular diseases . Cerebrovascular diseases

\section{Introduction}

Nonsteroidal anti-inflammatory drugs (NSAIDs), including low-dose aspirin (LDA), are known to disrupt the mucosal resistance to gastric acid through mechanisms including the decreased production of endogenous prostaglandin in the gastric mucosa, and are thus associated with adverse events such as gastric or duodenal ulcers. In one Japanese study of patients presenting with a bleeding ulcer, $7.6 \%$ were taking LDA [1]. Another study found the point prevalence of ulcers in LDA users to be 11.9-15.2\%, irrespective of the aspirin formulation [2]. Furthermore, several observational studies have suggested that the increasing use of LDA is becoming a major cause of bleeding ulcers [3]. In a Japanese single-institution report, ulcer lesions were endoscopically identified in 38 (12.4\%) of 305 patients taking LDA [4].

When patients present with gastrointestinal bleeding, discontinuation of LDA is recommended according to various guidelines [5]. However, discontinuation of LDA can be associated with a recurrence of disease, and this can result in serious outcomes [6]. Thus, it is vitally important to ensure prophylaxis of gastric or duodenal ulcers in patients on LDA therapy.

In this context, a number of controlled studies have reported on the prevention of gastric or duodenal ulcers

\footnotetext{
K. Shimada

Division of Cardiovascular Medicine,

Department of Internal Medicine, Jichi Medical University

Hospital, 3311-1 Yakushiji, Shimotsuke,

Tochigi 329-0498, Japan

S. Uchiyama

Department of Neurology, Tokyo Women's Medical University,

Kawada-cho, Shinjuku-ku, Tokyo 162-8666, Japan

N. Uemura

Department of Gastroenterology, Kohnodai Hospital, National Center for Global Health and Medicine,

Kohnodai, Ichikawa, Chiba 272-8516, Japan
}

with regular-dose $\mathrm{H} 2$-receptor antagonists or proton pump inhibitors (PPIs) in patients during LDA therapy [7-10]. Based on the evidence obtained to date, a clinical expert consensus statement [5] recommends PPIs as the preferred agents for the prophylaxis of LDA-associated gastrointestinal injury. However, to date, low-dose lansoprazole has not been evaluated in a clinical trial for its prophylactic efficacy in patients with definitive evidence of previous ulcer development.

This study thus aimed to examine the preventive effect of low-dose lansoprazole ( $15 \mathrm{mg}$ daily) against the recurrence of gastric or duodenal ulcer associated with longterm LDA therapy in patients with definitive evidence of previous ulcer history. Ulcer recurrence was defined as endoscopically confirmed ulcers, and the occurrences of gastric or duodenal bleeding with or without hospitalization were also evaluated. Appendix 1 shows the list of investigators for the Lansoprazole Ulcer Prevention Study Group (low-dose aspirin therapy).

Given that no drug has been proven to be effective for the prevention of gastric or duodenal ulcer associated with LDA therapy in Japan, and given that it is unethical to conduct a placebo-controlled trial in patients at high risk of developing gastric or duodenal ulcers, the present study was designed to compare the efficacy of lansoprazole $15 \mathrm{mg}$ once daily and gefarnate $50 \mathrm{mg}$ twice daily [11, 12]. Gefarnate is a cytoprotective anti-ulcer agent which is approved for the treatment for gastric or duodenal ulcers. These cytoprotective anti-ulcer agents are commonly prescribed as prophylactic drugs to reduce NSAID- or LDAinduced gastrointestinal injury, although they have not been investigated in a controlled trial for the latter indication.

\section{Methods}

\section{Design overview}

The study protocol was approved by the Ethics Committee of each participating institution, and all patients gave written informed consent to participate in the study. The Independent Data Monitoring Committee planned an interim analysis in advance to investigate whether or not to continue the study on the basis of interim efficacy and safety findings, based on the predefined criteria. An independent statistician performed the interim analysis on behalf of the Independent Data Monitoring Committee. After the committee made the decision to discontinue the double-blind trial, the patients at the 68 participating healthcare institutes were invited to move on to the followup trial with open-label lansoprazole treatment lasting up to 6 months. This trial was registered with ClinicalTrials.gov, number NCT00762359. 
Setting and participants

Patients were enrolled in the study if they met the following criteria: those who were being given LDA when they gave informed consent, and who required long-term LDA therapy after the start of the study (day 1) with the investigational drug; and those in whom a history of gastric or duodenal ulcer (or gastroduodenal ulcer) was confirmed by endoscopy, i.e., those who were confirmed to have an ulcer scar on day 1 or were confirmed to have an ulcer or ulcer scar in an endoscopic examination performed prior to day 1 (e.g., photographs, films).

Patients were excluded if they were confirmed to have an open gastric or duodenal ulcer or an active upper gastrointestinal hemorrhage by endoscopy on day 1; aspirininduced asthma or hypersensitivity to NSAIDs including aspirin, or a history of hypersensitivity; a history of surgery or a planned operation which affects gastric secretion (e.g., upper gastrointestinal tract resection, vagotomy); clinically significant liver or kidney disorder (including liver tests demonstrating aspartate aminotransferase [AST]/alanine aminotransferase [ALT] values 2.5 times or higher than their upper limit of normal, or creatinine levels 2.0 times or higher than its upper limit of normal); or an active cancer.

All patients confirmed to be eligible at each trial site were reassessed for their eligibility, based either on endoscopic images on films or data submitted after randomization, by an independent panel of expert endoscopists.

\section{Randomization and intervention}

Patients who met the inclusion criteria were randomly assigned to one of the following two treatment groups: a group receiving the investigational drug (lansoprazole $15 \mathrm{mg}$ orally given once daily) and the cytoprotective anti-ulcer agent, gefarnate placebo (twice daily) or a group receiving gefarnate $[11,12](50 \mathrm{mg}$ orally given twice daily) and a lansoprazole placebo (once daily), in combination with LDA (81-324 mg given once daily) for a duration of 12 months or longer (up to 30 months). Lansoprazole and gefarnate placebos were used to ensure that all patients followed the same regimen and that blinding was maintained. The treatment-group assignment was done by computer-generated random sequence numbers. Patients were randomly assigned by investigators to receive lansoprazole or gefarnate at a 1:1 ratio according to the unique sequential numbers for the study drugs, which were pre-assigned to each study site before the start of the treatment. When the onset of ulcer was diagnosed endoscopically or LDA was changed to different drugs, the subjects were excluded from the study at that time point.
Outcomes and measurements

The primary endpoint was the recurrence of gastric or duodenal ulcers, defined as patients confirmed to have active-stage or healing-stage ulcers associated with a mucosal defect with whitish exudates measuring $3 \mathrm{~mm}$ or greater. All ulcers confirmed on endoscopy and reported from each study site were reconfirmed by the independent expert panel, based on submitted films. The secondary endpoints were the development of gastric and/or duodenal hemorrhagic lesions as observed with endoscopy, treatment discontinuations due to lack of efficacy, gastric and/or duodenal mucosal damage as assessed with a modified Lanza score [13], and gastrointestinal symptoms.

\section{Follow-up procedures}

Endoscopy was scheduled every 12 weeks until 12 months of treatment and every 24 weeks after 12 months. Nonscheduled endoscopy was also performed if patients were suspected of having symptoms associated with ulcers or signs and symptoms indicative of gastrointestinal bleeding.

Every 4 weeks, clinical laboratory tests (chemistry, hematology, and urinalysis) were performed, blood pressure was measured, compliance checks (returned tablet counts) were conducted, and patients were asked about any adverse effects they experienced. All patients were scheduled to receive the study treatments in a double-blind fashion until 12 months after the start of the study in the last enrolled patient. After the termination of the double-blind trial, patients at the 68 study sites were invited to participate in the follow-up study, in which all patients were treated once daily with lansoprazole $15 \mathrm{mg}$. If onset of an ulcer was confirmed on endoscopy in a patient, the patient discontinued their medication, and antiulcer treatment - such as full-dose PPI therapy - was offered for ulcer healing.

\section{Statistical analysis}

The 1-year cumulative incidences of ulcer events in patients treated with lansoprazole and gefarnate, in addition to LDA therapy, were assumed to be 6 and 13\%, respectively, which suggested that the hazard ratio (HR) of the lansoprazole-treated group relative to the gefarnatetreated group was 0.44 under an exponential assumption of event distributions. We required a total of 64 ulcer events (endpoints) for the two treatment groups to ensure a statistical power of $90 \%$ using a log-rank test with a two-sided alpha of 5\%. To observe 64 events, we required the enrollment of 406 patients for each treatment group at randomization, for a total of 812 patients for the study, assuming a mean follow-up duration of 1 year and a 1-year dropout rate of $20 \%$. 
One interim analysis was planned in advance for the Independent Data Monitoring Committee to perform when half of the required number of ulcer events was observed. The O'Brien-Fleming boundary, based on the information fraction of 0.5, was employed for an overall significance level of $\alpha=0.05$. To avoid unnecessary trial hazard to subjects assigned to either arm, we planned to discontinue the doubleblind trial if the difference in the primary endpoint reached significance at $P=0.0038$ at the interim analysis.

The cumulative incidences of the primary and secondary endpoints were estimated by using the Kaplan-Meier method and compared between the treatment groups by using the log-rank test. For event-free cases the event times were censored either at the point of the last endoscopy performed or at the point of early withdrawal. We also performed multivariate Cox regression analyses to adjust for possible effects of baseline variables on event times. The final analyses were conducted for the full-analysis set (FAS), defined as all patients who were randomized and received one or more doses of the study medication. In the survival analysis, the patients at risk were defined as all event-free FAS patients who had at least one post-randomization assessment with endoscopy.

Differences in adverse events between the lansoprazole and gefarnate groups were tested for significance by using the $\chi^{2}$ test.

Analyses were conducted using SAS software (version 9.1.3; SAS Institute, Cary, NC, USA). One and the same statistician (S.M.) had full access to all the trial data and conducted statistical analyses independently of the sponsoring company.

\section{Role of the funding source}

Takeda Pharmaceutical Company Limited (the Sponsor) and its contractor provided all financial and material support for the study design, data collection, data analysis, data interpretation, and preparation and review of manuscripts. The Sponsor was also responsible for consultations with the authors and the members of this study group about the study design and about monitoring of the study. The principal investigator (K.S.) was responsible for the study design and for preparation of the manuscript. All coauthors reviewed the manuscript, and necessary revisions were made to accommodate their suggestions and opinions.

\section{Results}

Study patients

This prospective, double-blind, randomized, active-controlled trial with an open-label 6-month follow-up study was conducted at a total of 94 healthcare institutions in Japan, in accordance with the principles of good clinical practice and the Declaration of Helsinki. The Independent Data Monitoring Committee performed an interim analysis, based on data that had become available from 414 patients. The cumulative number of ulcer events at the interim analysis was 30 in the gefarnate group and three in the lansoprazole group. The HR was estimated as $0.080(95 \%$ CI 0.023-0.264; $P<0.001,2$-sided log-rank test), verifying the efficacy of lansoprazole compared with gefarnate and, accordingly, the Independent Data Monitoring Committee made the decision to terminate the initial part of the study early. Data completion and analysis were performed based on data collected at the termination of the trial. The results discussed here are based on the final data.

Figure 1 shows the flow diagram of this trial. Of the 771 patients enrolled, 461 patients were randomized, while the remaining 310 patients were excluded, primarily because they were not confirmed to have evidence of past gastric or duodenal ulcers on baseline endoscopy after enrollment. Of the 461 patients randomized, 226 were assigned to receive lansoprazole and 235 to receive gefarnate. Of the 235 patients assigned to gefarnate, the study medication was not given to one patient, because malignancy was found in this patient (a violation of the study protocol). Thus, the FAS population comprised a total of 460 patients, with 226 and 234 patients in the lansoprazole group and the gefarnate group, respectively. The numbers of withdrawals were similar in the treatment groups, with 59 withdrawals $(26.1 \%)$ in the lansoprazole group and 57 (24.3\%) in the gefarnate group. The most frequent reasons for withdrawal were adverse events, which occurred in 20 (33.9\%) patients in the lansoprazole group and $23(40.4 \%)$ patients in the gefarnate group; followed by protocol deviations (including failure to take the medication) and consent withdrawals in $21(35.6 \%)$ and $13(22.0 \%)$ patients in the lansoprazole group, and $13(22.8 \%)$ and $10(17.5 \%)$ patients, respectively, in the gefarnate group. Additionally, four patients in the gefarnate group withdrew due to lack of efficacy or suspected ulcer-related symptoms/diagnoses. The median duration of follow-up was 7.5 months (range 0.1-17.0) for the lansoprazole group and 5.7 months (range 0.0-16.5) for the gefarnate group. Compliance with the study medication and LDA therapy was similarly high in the two treatment groups. There was no difference between the treatment groups in the frequency distribution of baseline variables (Table 1).

\section{Efficacy}

In the FAS population, the cumulative number of gastric or duodenal ulcer recurrences, i.e., the primary endpoint, at the end of the study was $6 / 226(2.7 \%)$ in the lansoprazole 
Fig. 1 Patient disposition in this trial (2010 CONSORT flow diagram)

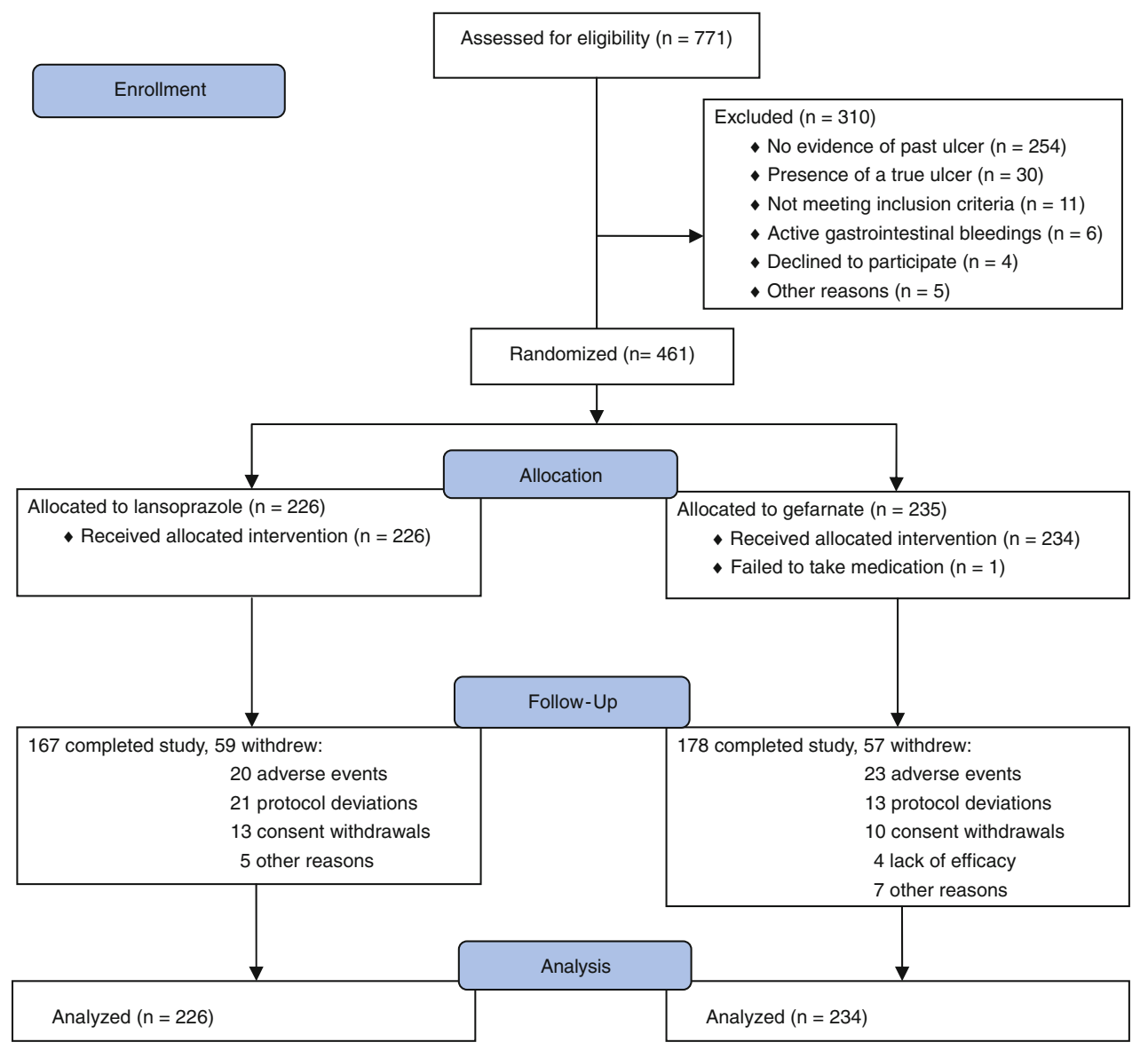

group and 53/234 (22.6\%) in the gefarnate group (Table 2). The cumulative recurrences on days 91, 181, and 361 from the start of the study were estimated as $1.5 \%$ (95\% CI $0.00-3.20), 2.1 \%$ (95\% CI $0.06-4.08$ ), and $3.7 \%$ (95\% CI 0.69-6.65), respectively, for the lansoprazole group, compared to $15.2 \%$ (95\% CI 10.17-20.22), $24.0 \%$ (95\% CI 17.84-30.21), and 31.7\% (95\% CI 23.86-39.57), respectively, for the gefarnate group. The HR of the lansoprazole group relative to the gefarnate group was estimated as 0.099 (95\% CI $0.042-0.230$ ) - $90.1 \%$ risk reduction, and the difference was highly significant (log-rank test, $P<0.001$ ) (Table 2; Fig. 2).

As to the secondary endpoints (Table 2), the risk of developing gastric/duodenal ulcers or hemorrhagic lesions in the lansoprazole group was significantly lower than that in the gefarnate group (log-rank test, $P<0.001$ ). Similarly, the risk of having gastric/duodenal ulcers, hemorrhagic lesions, or treatment discontinuations due to lack of efficacy was significantly lower in the lansoprazole group than in the gefarnate group (log-rank test, $P<0.001$ ).

The magnitude of risk reduction for gastric or duodenal ulcers (primary endpoint) was generally stable for all subgroups as defined by each baseline variable (Table 3). The analyses in both Helicobacter pylori-positive and -negative subgroups showed ulcer risk reductions, with an
HR of 0.061 (95\% CI $0.019-0.197 ; P<0.001)$ and an HR of 0.206 (95\% CI $0.060-0.710 ; P=0.02$ ), respectively, in each of the subgroups in the lansoprazole group as compared to the gefarnate group. Furthermore, the risk reduction, in terms of HR, was estimated as $0.085(95 \%$ CI $0.034-0.216 ; P<0.001$ by a Wald test) after adjustment for the baseline variables, H. pylori status, CYP2C19 polymorphism, age, gender, smoking, alcohol consumption, and concomitant use of anticoagulants in a multivariate Cox regression analysis (Table 4).

We also analyzed the sites of the recurrent ulcers to examine whether the ulcer recurred at sites similar to those of the scars observed at the start of the study. Whitish or red scars were reported in 397 patients ( $86.1 \%$ of total). We further obtained data on the location of the scars from all 59 patients in whom ulcers had relapsed. In $36(61.0 \%)$ of these patients, ulcer recurrence was observed at sites similar to those of the scars seen at the start of the study.

Gastrointestinal damage, as assessed by a modified Lanza score [13], from the start of treatment tended to improve in the lansoprazole group, but to worsen in the gefarnate group, throughout the course of treatment (Supplemental Fig. 1).

In the FAS population, the cumulative number of patients who developed gastric or duodenal hemorrhagic 
Table 1 Demographic and baseline characteristics of Japanese patients randomized to treatment

Data are numbers (and \% of total) except where otherwise indicated

LDA low-dose aspirin, $E M$

Extensive metabolizers, $P M$ poor metabolizers

a Those who reported taking LDA for $>3$ years prior to the start of the study medication were construed as having taken it for 3 years

b Some patients were included in more than 1 disease category. The category "Others" includes treatments such as carotid arteriosclerosis or carotid artery occlusion

c Unknown in 1 patient

${ }^{d}$ Unknown in 46 patients for whom consent was not obtained for the CYP2C19 polymorphism test

\begin{tabular}{|c|c|c|}
\hline & Lansoprazole $(n=226)$ & Gefarnate $(n=235)$ \\
\hline Mean age (SD), years & $69.3(8.57)$ & $68.7(8.79)$ \\
\hline \multicolumn{3}{|l|}{ Sex } \\
\hline Males & 175 (77.4) & $192(81.7)$ \\
\hline Females & $51(22.6)$ & $43(18.3)$ \\
\hline Current smoking status & $52(23.0)$ & $53(22.6)$ \\
\hline Alcohol consumption & $102(45.1)$ & $123(52.3)$ \\
\hline Mean duration (SD) of prior LDA (months) ${ }^{\mathrm{a}}$ & $25.4(13.34)$ & $24.9(13.54)$ \\
\hline \multicolumn{3}{|l|}{ Status of concomitant aspirin use } \\
\hline Aspirin dialminate & $27(11.9)$ & $28(11.9)$ \\
\hline $81 \mathrm{mg}$ & $26(11.5)$ & $26(11.1)$ \\
\hline $162 \mathrm{mg}$ & $1(0.4)$ & $3(1.3)$ \\
\hline Aspirin & $199(88.1)$ & $207(88.1)$ \\
\hline $100 \mathrm{mg}$ & $193(85.4)$ & $194(82.6)$ \\
\hline $200 \mathrm{mg}$ & $7(3.1)$ & $13(5.5)$ \\
\hline \multicolumn{3}{|l|}{ Underlying disease $^{\mathrm{b}}$} \\
\hline Ischemic heart disease & $109(48.2)$ & $120(51.1)$ \\
\hline Ischemic stroke & $96(42.5)$ & $97(41.3)$ \\
\hline Others & $50(22.1)$ & $49(20.9)$ \\
\hline \multicolumn{3}{|l|}{ H. pylori status ${ }^{\mathrm{c}}$} \\
\hline Positive & $137(60.6)$ & $125(53.2)$ \\
\hline Negative & $89(39.4)$ & $109(46.4)$ \\
\hline \multicolumn{3}{|l|}{ CYP2C19 polymorphism ${ }^{\mathrm{d}}$} \\
\hline EM & $163(72.1)$ & $181(77.0)$ \\
\hline PM & $40(17.7)$ & $34(14.5)$ \\
\hline \multicolumn{3}{|l|}{ Mean compliance rate (SD), \% } \\
\hline Study drug & $99.03(2.268)$ & $98.17(7.073)$ \\
\hline LDA therapy & $93.84(3.319)$ & $93.12(7.400)$ \\
\hline
\end{tabular}

Table 2 Effect of lansoprazole on each component of the primary and secondary endpoints

\begin{tabular}{|c|c|c|c|c|}
\hline & $\begin{array}{l}\text { Lansoprazole }^{\mathrm{a}} \\
(n=226)\end{array}$ & $\begin{array}{l}\text { Gefarnate }^{\mathrm{b}} \\
(n=234)\end{array}$ & $\begin{array}{l}\text { Hazard ratio } \\
(95 \% \mathrm{CI})\end{array}$ & $P$ value ${ }^{\mathrm{c}}$ \\
\hline Number at risk at baseline $^{\mathrm{d}}$ & 213 & 227 & & \\
\hline \multicolumn{5}{|l|}{ Primary endpoint } \\
\hline Gastric or duodenal ulcer & 6 & 53 & $0.099(0.042-0.230)$ & $<0.001$ \\
\hline \multicolumn{5}{|l|}{ Secondary endpoints } \\
\hline Gastric/duodenal ulcer or hemorrhagic lesion & 7 & 56 & $0.109(0.050-0.239)$ & $<0.001$ \\
\hline $\begin{array}{l}\text { Gastric/duodenal ulcer, hemorrhagic lesion or } \\
\text { treatment discontinuation due to lack of efficacy }\end{array}$ & 7 & 59 & $0.104(0.047-0.228)$ & $<0.001$ \\
\hline \multicolumn{5}{|l|}{ Component } \\
\hline Gastric ulcer & 6 & 40 & & \\
\hline Duodenal ulcer & 0 & 15 & & \\
\hline Hemorrhagic lesion & 2 & 9 & & \\
\hline Treatment discontinuation due to lack of efficacy & 0 & 4 & & \\
\hline
\end{tabular}

CI confidence interval

${ }^{\text {a }}$ Patients received lansoprazole $15 \mathrm{mg}$ daily

${ }^{\mathrm{b}}$ Patients received gefarnate $50 \mathrm{mg}$ twice daily

${ }^{c}$ Log-rank test

d The number of patients at risk included all full-analysis set patients who received at least 1 endoscopy assessment post-randomization, and had no acute-stage or healing-stage gastric or duodenal ulcer as confirmed by the Independent Adjudication Committee 
Fig. 2 Kaplan-Meier estimates of the cumulative incidence of gastric or duodenal ulcers and hemorrhagic lesions in the treatment groups
Table 3 Analysis of subgroups as defined by each baseline variable

Data are $n /$ at risk; at risk: the number of patients at risk included all full-analysis set patients who had at least 1 postrandomization endoscopy assessment, and had no acutestage or healing-stage gastric or duodenal ulcer as confirmed by the Independent Adjudication Committee

$E M$ Extensive metabolizers, $P M$ Poor metabolizers

a, b, d Results of Cox regression analyses; hazard ratio $(95 \% \mathrm{CI})$, and $P$ value: ${ }^{\text {a }} 0.6746$

(0.1361-3.3426), $P=0.63$;

b 2.2665 (1.2451-4.1258),

$P=0.01 ;{ }^{\mathrm{d}} 0.6970$

(0.3373-1.4405), $P=0.33$

c Hazard ratio relative to these subgroups could not be estimated

e Could not be estimated

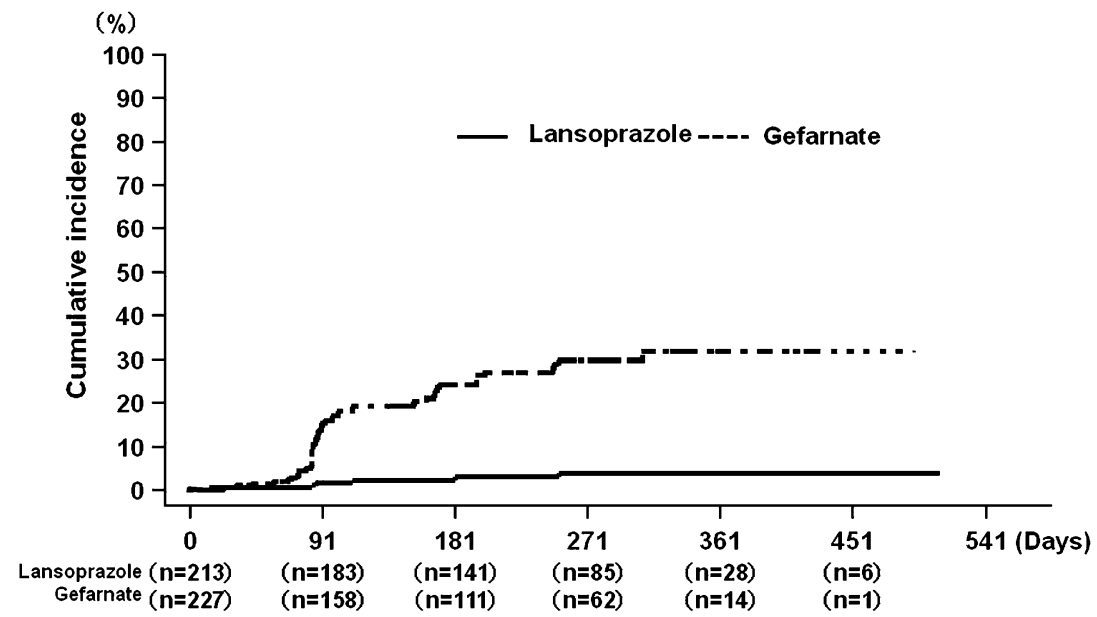

Baseline characteristics Recorded number of patients with gastric or Cox regression analysis duodenal ulcer

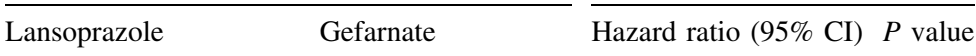

H. pylori status

Positive

$3 / 128^{\mathrm{a}}$

Negative

$3 / 85^{\mathrm{a}}$

CYP2C19

$\mathrm{PM}$

EM

Age

32-64 years

65-88 years

$5 / 156$

$0 / 38^{\mathrm{c}}$

$5 / 155^{\mathrm{c}}$

Gender

Male

Female

Smoker

Yes 1/49

No

$5 / 164$

Alcohol consumption

$\begin{array}{ll}\text { Yes } & 4 / 96 \\ \text { No } & 2 / 117\end{array}$

Concomitant use of anticoagulants

$\begin{array}{lll}\text { Yes } & 2 / 47 & 19 / 69 \\ \text { No } & 4 / 166 & 34 / 158\end{array}$

$38 / 122^{\mathrm{b}}$
$15 / 105^{\mathrm{b}}$
$9 / 33^{\mathrm{d}}$
$39 / 175^{\mathrm{d}}$
$14 / 72$
$39 / 155$
$43 / 184$
$10 / 43$
$16 / 51$
$37 / 176$
$24 / 120$
$29 / 107$
$19 / 69$
$34 / 158$

$0.061(0.019-0.197)$

$<0.001$

$0.206(0.060-0.710)$

0.02

$0.000\left(0.000\right.$ to $\left.-^{\mathrm{e}}\right) \quad-$

$0.125(0.049-0.317)<0.001$

$0.072(0.009-0.550) \quad 0.02$

$0.106(0.042-0.268)<0.001$

$0.104(0.041-0.264)<0.001$

$0.082(0.011-0.643) \quad 0.02$

$0.048(0.006-0.365) \quad 0.01$

$0.122(0.048-0.311)<0.001$

$0.170(0.059-0.491) \quad 0.01$

$0.052(0.012-0.219) \quad<0.001$

$0.127(0.029-0.546) \quad 0.01$

$0.091(0.032-0.256)<0.001$ lesions at the end of the study was two of the 226 patients in the lansoprazole group versus nine of the 234 patients in the gefarnate group. The cumulative incidence rate was calculated by the Kaplan-Meier method (Supplemental Fig. 2) and the risk of hemorrhage was shown to be significantly lower in the lansoprazole group than in the gefarnate group. Bleeding ulcers occurred in one patient in the lansoprazole group and five in the gefarnate group. Other gastric or duodenal bleeding was primarily related to erosions. The number of patients who were hospitalized with serious adverse events leading to gastric or duodenal bleeding was one in the lansoprazole group and five in the gefarnate group.

Of the 460 patients randomized to lansoprazole or gefarnate in this trial, 262 who had received lansoprazole or gefarnate were included in an open-label follow-up trial to examine the outcome after another 24 weeks of treatment with lansoprazole, in addition to LDA. During this openlabel follow-up trial period, no gastric or duodenal ulcer recurrence was observed in the study participants 
Table 4 Results of multivariate Cox regression analysis using baseline variables
EM Extensive metabolizers, $P M$ Poor metabolizers

\begin{tabular}{lllc}
\hline Baseline characteristics & Direction estimation & Multivariate analysis & \\
\cline { 3 - 4 } & & Hazard ratio (95\% CI) & $P$ value \\
\hline Treatment group & Lansoprazole/gefarnate & $0.085(0.034-0.216)$ & $<0.001$ \\
H. pylori status & Positive/negative & $2.057(1.137-3.720)$ & 0.02 \\
CYP2C19 & PM/EM & $1.434(0.668-3.076)$ & 0.36 \\
Age & 10 years' increase & $1.459(1.045-2.036)$ & 0.03 \\
Gender & Male/female & $0.893(0.437-1.823)$ & 0.76 \\
Smoking status & Yes/no & $1.532(0.820-2.863)$ & 0.19 \\
Alcohol consumption & Yes/no & $1.047(0.588-1.866)$ & 0.88 \\
Concomitant use of anticoagulants & Yes/no & $1.200(0.665-2.166)$ & 0.55 \\
\hline
\end{tabular}

(Supplemental Table 1). Importantly, compared to the ulcer recurrence in the double-blind phase, no ulcer recurrence was observed from the gefarnate group during the 6 months of the lansoprazole open study, indicating the potent preventive effect of lansoprazole.

\section{Adverse events}

With respect to adverse events observed in the doubleblind study period (Table 5), diarrhea was noted significantly more frequently in the lansoprazole group than in the gefarnate group, while reflux esophagitis occurred significantly more frequently in the gefarnate group. No serious adverse drug reactions occurred in the lansoprazole group, versus one (liver disorder) in the gefarnate group. No deaths occurred in either group. Of the 21 patients who discontinued lansoprazole, four were suspected of having possible adverse drug reactions, which included stomatitis, abnormal liver function tests, diarrhea, constipation, and palpitation. In the gefarnate group, six patients experienced possible adverse drug reactions, which included dyspepsia, Mallory-Weiss syndrome, eczema, tinnitus, toxic skin eruption, and liver disorder.

From the start of the double-blind study through the continued follow-up trial, four bone fractures were observed in four patients in the lansoprazole group, with three events occurring during the double-blind trial period $(P=0.08$, $\chi^{2}$ test, vs. gefarnate group), and the other one event occurring in the open-label follow-up period. Investigators reported the causes of the bone fractures to be factors such as aging, accidental fall occurring as a result of a subject's inattentiveness, and the like; hence, their causal relationship to lansoprazole was denied. No bone fracture occurred in the gefarnate group during the double-blind period.

During the entire study, including the follow-up trial, two deaths occurred, due to ventricular fibrillation and acute myocardial infarction, respectively; their causal relationship to lansoprazole was denied by the investigators. Serious adverse reactions occurred in 51/339 (15.0\%) patients, of which 26 occurred in the follow-up trial.
Of these, melena occurred in one patient $(0.3 \%)$ and this was the only event whose causal relationship to lansoprazole could not be denied. Thirty-nine treatment discontinuations occurred in the entire period; of these, 16 occurred in the follow-up period, where the most common event was diarrhea, which occurred in four patients $(1.2 \%)$.

\section{Discussion}

Given that no drug has been proven to be effective for the prevention of gastric or duodenal ulcer associated with LDA therapy in Japan, and given that it is unethical to conduct a placebo-controlled trial in patients at high risk of developing gastric or duodenal ulcers, the present study was designed to compare the efficacy of lansoprazole $15 \mathrm{mg}$ once daily and gefarnate $50 \mathrm{mg}$ twice daily $[11,12]$. Gefarnate is a cytoprotective anti-ulcer agent which is approved for the treatment of gastric or duodenal ulcers. These cytoprotective anti-ulcer agents are commonly prescribed as prophylactic drugs to reduce NSAID- or LDA-induced gastrointestinal injury, although they have not been investigated in a controlled trial for the latter indication.

To minimize risks to the patients enrolled in this trial, they were strictly assessed by endoscopic examination for eligibility. In addition, unlike most long-term clinical trials conducted to date in a similar patient population, frequent endoscopic examinations (every 3 or 6 months) were scheduled by the protocol to closely monitor the study subjects for early detection of ulcer recurrence.

While there are arguments for and against $H$. pylori eradication in long-term NSAID users [14], one study showed that $H$. pylori eradication prior to LDA therapy was equivalent to omeprazole therapy in preventing recurrent gastrointestinal bleeding [15], although the study was underpowered to demonstrate such equivalence. However, in other studies, the ulcerogenic effect of LDA was not abolished by $H$. pylori eradication in high-risk patients [7], and $20 \%$ of an entire cohort of patients who had developed dyspeptic or bleeding ulcers/erosions during prophylactic 
Table 5 Frequency of adverse events

\begin{tabular}{|c|c|c|c|}
\hline Adverse events that occurred in the double-blind period & Lansoprazole $(n=226)$ & Gefarnate $(n=234)$ & $P$ value \\
\hline All adverse events & $166(73.5)$ & $168(71.8)$ & 0.70 \\
\hline Causal relationship to drug not deniable & $26(11.5)$ & $25(10.7)$ & 0.78 \\
\hline Leading to discontinuations & $21(9.3)$ & $24(10.3)$ & 0.73 \\
\hline Serious adverse events & $27(11.9)$ & $26(11.1)$ & 0.78 \\
\hline Causal relationship to drug not deniable & $0(0.0)$ & $1(0.4)$ & 0.33 \\
\hline Deaths & $0(0.0)$ & $0(0.0)$ & - \\
\hline \multicolumn{4}{|c|}{ Adverse events reported in at least $3 \%$ of the total in each group } \\
\hline Nasopharyngitis & $54(23.9)$ & $55(23.5)$ & 0.93 \\
\hline Constipation & $14(6.2)$ & $8(3.4)$ & 0.17 \\
\hline Fall & $13(5.8)$ & $9(3.8)$ & 0.34 \\
\hline Diarrhea & $19(8.4)$ & $2(0.9)$ & $<0.001$ \\
\hline Reflux esophagitis & $3(1.3)$ & $16(6.8)$ & 0.01 \\
\hline Back pain & $10(4.4)$ & $5(2.1)$ & 0.17 \\
\hline Elevated blood creatine phosphokinase levels & $7(3.1)$ & $8(3.4)$ & 0.85 \\
\hline Eczema & $5(2.2)$ & $7(3.0)$ & 0.61 \\
\hline Hypertension & $7(3.1)$ & $3(1.3)$ & 0.19 \\
\hline
\end{tabular}

Adverse events reported in patients who received lansoprazole throughout the double-blind and open-label study

\begin{tabular}{lc}
\hline All adverse events & $279(82.3)$ \\
Causal relationship to drug not deniable & $55(16.2)$ \\
Leading to discontinuations & $39(11.5)$ \\
Serious adverse events & $51(15.0)$ \\
Causal relationship to drug not deniable & $1(0.3)$ \\
Deaths & $2(0.6)$ \\
Adverse events reported in at least 3\% of the total in each group & $113(33.3)$ \\
Nasopharyngitis & $32(9.4)$ \\
Diarrhea & $23(6.8)$ \\
Constipation & $19(5.6)$ \\
Fall & $17(5.0)$ \\
Hypertension & $16(4.7)$ \\
Elevated blood creatine phosphokinase levels & $16(4.7)$ \\
Back pain &
\end{tabular}

Table data are numbers $(\%)$ of patients in whom an event occurred at least 1 time during the trial

treatment with famotidine were all found to be $H$. pylorinegative [10]. Therefore, trial results reported to date are inconsistent, although $H$. pylori eradication is generally recommended in most situations [14, 16]. Besides, because it was difficult to predict the influence of rebound acid hypersecretion occurring after $H$. pylori eradication [17] on the results of the present study, the subjects who required long-term LDA therapy were not obliged to undergo H. pylori eradication prior to administration of the study drug, and the protocol was designed to allow patients to be treated with a PPI or $H$. pylori eradication until the day immediately before the start of treatment with lansoprazole or gefarnate, given the varying durations of prior LDA use among the patients. Thus, the study attempted to evaluate the efficacy of lansoprazole vs. gefarnate against ulcer recurrences in an ordinary clinical setting, in which H. pylori eradication was implemented at the discretion of the attending physician.

Analyses in both $H$. pylori-positive and -negative subgroups in the present study showed ulcer risk reductions in the lansoprazole group as compared to the gefarnate group, although the risk reduction rate was higher in the H. pyloripositive patients. This finding is consistent with a previous study in patients at relatively low risk for ulcer complications [8] and supports the usefulness of low-dose lansoprazole in Japan, where the prevalence of $H$. pylori infection is high [18]. Additionally, although more $H$. pylori-negative patients will need prophylactic treatment for preventing 
LDA ulcers in Japan, where the $H$. pylori infection rate is predicted to gradually decrease [19], low-dose lansoprazole should still be effective in these patients as well.

A final analysis of the study data showed that lansoprazole produced a $90.1 \%$ reduction in the risk of ulcer recurrence, which was highly significant. The reduction rate is similar to that in a placebo-controlled study conducted in Hong Kong in patients taking LDA [7] and even higher than that in another study in patients with ulcers associated with LDA, where the rate of risk reduction was found to be about $70 \%$ [8].

Although the recurrence of ulcers observed by endoscopy was assessed as the primary endpoint in the present study, other clinical endpoints, such as gastrointestinal bleeding and patient hospitalization, were also compared between the treatment groups, because these true clinical outcomes are very important in evaluating the drugs for efficacy. In this study, more patients in the gefarnate group developed gastric or duodenal hemorrhagic lesions and were hospitalized with serious adverse events leading to gastric or duodenal bleeding. Thus, overall, lansoprazole was superior to gefarnate in all endpoints assessed in this study.

Furthermore, there were no new-onset ulcers noted in the additional 6 months' follow-up trial, supporting the idea that lansoprazole provides superior long-term efficacy in preventing LDA-associated gastric/duodenal ulcers, compared to gefarnate.

Of note, the present study represents the longest followup (18 months or more) of patients with a definite history of gastric or duodenal ulcer who required long-term LDA therapy, of all reports (3-12 months) published in the literature [7-10].

Thus, lansoprazole appears to have an important role to play in reducing the risk of gastroduodenal ulcers in patients at high risk of developing ulcers who require long-term LDA therapy due to cardiovascular and cerebrovascular disease, while at the same time allowing such antiplatelet therapy to reduce thromboembolic events in these patients.

Acknowledgments Grant support by Takeda Pharmaceutical Company Limited.

Open Access This article is distributed under the terms of the Creative Commons Attribution Noncommercial License which permits any noncommercial use, distribution, and reproduction in any medium, provided the original author(s) and source are credited.

\section{Appendix 1: Investigators in the Lansoprazole Ulcer Prevention Study Group (low-dose aspirin therapy)}

Tetsuya Noto, Hakodate Kyouritsu Medical Corporation, Hakodate, Hokkaido, Japan; Masanori Murakami,
Hokkaido PWFAC Asahikawa Kosei General Hospital, Asahikawa, Hokkaido, Japan; Hiroshi Kozawa, Hokkaido PWFAC Sapporo-Kosei General Hospital, Sapporo, Hokkaido, Japan; Eiji Araki, Sapporo Central Hospital, Sapporo, Hokkaido, Japan; Yukihiro Ibayashi, National Hospital Organization Hokkaido Cancer Center, Sapporo, Hokkaido; Kazuhiro Myoujin, National Hospital Organization Hokkaido Cancer Center, Sapporo, Hokkaido; Yoshimitsu Ishibashi, National Hospital Organization Hokkaido Cancer Center, Sapporo, Hokkaido; Fumitaka Shinojima, Hokkaido Health Coop Ryokuai Hospital, Sapporo, Hokkaido; Masao Yamauchi, Yamauchi Medical Clinic, Sapporo, Hokkaido; Katsunori Iijima, Tohoku University Hospital, Sendai, Miyagi; Tsuyoshi Shinozaki, Sendai Medical Center, Sendai, Miyagi; Hitoshi Sekine, Sendai City Hospital, Sendai, Miyagi; Yoshiaki Katahira, Tohoku Kosei-Nenkin Hospital, Sendai, Miyagi; Yasushi Matsumoto, Kohnan Hospital, Sendai, Miyagi; Masaharu Kanazawa, Sendai Open Hospital, Sendai, Miyagi; Masahiro Yagi, Sendai Cardiovascular Disease Center, Sendai, Miyagi; Mutsumi Nozue, Shonai Amakume Hospital, Amakume, Yamagata; Yuji Mizokami, Tokyo Medical University Ibaraki Medical Center, Kasumigauwa, Ibaraki; Shuichi Taguchi, Mito Medical Center, Mito, Ibaraki; Naoko Moriyama, Kasumigaura Medical Center, Kasumigaura, Ibaraki; Ikuo Kozakai, Kasumigaura Medical Center, Kasumigaura, Ibaraki; Gaku Ooki, Yuki Hospital, Yuki, Ibaraki; Takayoshi Yamanouchi, Mito General Hospital, Mito, Ibaraki; Hitoshi Tabata, Namegata District General Hospital, Namegata, Ibaraki; Takehiko Ayabe, Ayabe Medical Clinic, Hitachinaka, Ibaraki; Kentaro Sugano, Jichi Medical University Hospital, Shimotsuke, Tochigi; Hideki Nagaoka, Fujioka General Hospital Outpatient Center, Fujioka, Gunma; Syunroku Sugimoto, Sugimoto Hospital, Maebashi, Gunma; Tadahiko Kubo, Hanyu General Hospital, Hanyu, Gunma; Kazuyoshi Harano, Yotsukaido Tokushukai Medical Center, Yotsukaido, Chiba; Masahiro Yoshioka, Minamitama Hospital, Hachioji, Tokyo; Kazuhiko Segawa, National Hospital Organization Tokyo Hospital, Kiyose, Tokyo; Terunao Ashida, The Institute for Adult Disease, Marunouchi Hospital, Chiyoda-ku, Tokyo; Naomi Uemura, National Center for Global Health and Medicine, Shinjuku-ku, Tokyo; Masao Matsumoto, Tokyo Kosei Nenkin Hospital, Shinjuku-ku, Tokyo; Makoto Akaishi, Kitasato Institute Hospital, Minato-ku, Tokyo; Nobuyuki Matsuhashi, Nippon Telegraph and Telephone East Corporation Hospital, Shinagawa-ku, Tokyo; Hisakuni Sekino, Sekino Hospital, Toshima-ku, Tokyo; Jun Hashida, Eisei Clinic, Hachioji, Tokyo; Shigeto Kiyokawa, Fujimori Clinic, Hachioji, Tokyo; Yoshio Ohashi, Ekimae Building Clinic, Chuo-ku, Tokyo; Atsushi Takahashi, Yokosuka Kyosai Hospital, Yokosuka, Kanagawa; Takahiro Takei, Fujisawa 
Shounandai Hospital, Fujisawa, Kanagawa; Satoshi Kiyama, Chuo-Rinkan Hospital, Yamato, Kanagawa; Hitoshi Ohishi, Saiseikai Yokohama-shi Nanbu Hospital, Yokohama, Kanagawa; Sumihisa Abe, Koukan Clinic, Kawasaki, Kanagawa; Muneatsu Toshima, Niitsu Medical Center Hospital, Niigata, Niigata; Tokio Shimomura, NHO Saigata National Hospital, Joetsu, Niigata; Masatoshi Ikeda, Kanazawa Cardiovascular Hospital, Kanazawa, Ishikawa; Kousei Ueda, Komatsu Municipal Hospital, Komatsu, Ishikawa; Kouji Shinmura, Shinmura Hospital, Shiroyama, Ishikawa; Kazuo Satake, Fukui General Hospital, Fukui, Fukui; Toshiyuki Noda, Gifu Prefectural General Medical Center, Gifu, Gifu; Masatoshi Koshiji, Gifu Central Hospital, Gifu, Gifu; Takuya Umemoto, National Hospital Organization Shizuoka Medical Center, Shunto-gun, Shizuoka; Mashio Nakamura, Mie University Hospital, Tsu, Mie; Makoto Kuroda, Matsusaka City Hospital, Matsusaka, Mie; Hitoshi Yoshimura, Mie Prefectural Shima Hospital, Shima, Mie; Osamu Yamaoka, Social Insurance Shiga Hospital, Otsu, Shiga; Tateo Sugiyama, Kanai Hospital, Kyoto, Kyoto; Yasumasa Kondo, Horikawa Hospital, Kyoto, Kyoto; Eiji Ooyake, Uzumasa Hospital, Kyoto, Kyoto; Tsukasa Itabashi, Hokusetsu General Hospital, Takatsuki Osaka; Akio Saeki, Aino Hospital, Ibaraki, Osaka; Takeshi Morita, Matsubara Tokushukai Hospital, Matsubara, Osaka; Shinya Ohashi, Kitano Hospital, Osaka, Osaka; Shujiro Yazumi, Kitano Hospital, Osaka, Osaka; Kiyoshi Ashida, Osaka Saiseikai Nakatsu Hospital, Osaka, Osaka; Kenshi Fujii, Sakurabashi Watanabe Hospital, Osaka, Osaka; Kiyotaka Ookawa, Osaka Municipal General Medical Center, Osaka, Osaka; Takashi Abe, Osaka Police Hospital, Osaka, Osaka; Eitatsu Arai, Koyukai Nishinomiya Kyoritsu Neusurgical Hospital, Nishinomiya, Hyogo; Hiroyuki Ogawa, Nishinomiya Municipal Central Hospital, Nishinomiya, Hyogo; Hirofumi Okada, National Hospital Organization Hamada Medical Center, Hamada, Shimane; Hiroshi Mieno, Hiroshima General Hospital of the West Japan Railway Company, Hiroshima, Hiroshima; Hiroaki Oogoshi, Hiroshima City Asa Hospital, Hiroshima, Hiroshima; Shirou Okamoto, Kure Kyosai Hospital, Kure, Hiroshima; Koichi Noda, Higashihiroshima Medical Center, Hiroshima, Hiroshima; Yuji Furutani, National Hospital Organizaton Kanmon Medical Center, Shimonoseki, Yamaguchi; Shoichi Tanaka, Iwakuni Clinical Center, Iwakuni, Yamaguchi; Tomoki Inaba, Kagawa Prefectural Central Hospital, Takamatsu, Kagawa; Yuichiro Takagi, Takamatsu Hospital, Takamatsu, Kagawa; Akihiro Kojima, Takamatsu City Hospital, Takamatsu, Kagawa; Shin Kimoto, Juntenkai Hoshasen Daiichi Hospital, Imabari, Ehime; Mikio Yano, Yano Medical Clinic, Matsuyama, Ehime; Takashi Furuno and Katsuhito Yamamoto, Kochi Health Sciences Center, Kochi, Kochi; Naohiko Harada, National Hospital Organization Kyushu Medical Center,
Fukuoka, Fukuoka; Kyota Higashi, Fukuoka Wajiro Hospital, Fukuoka, Fukuoka; Shigekazu Nakano, Fukuoka Shin-Mizumaki Hospital, Onga-gun, Fukuoka; Yasuhiro Morikami, Kumamoto City Hospital, Kumamoto, Kumamoto; Yoshihiro Kimura, Kumamoto City Hospital, Kumamoto, Kumamoto; Kazuhiro Sugi, National Hospital Organization Kumamoto Medical Center, Kumamoto, Kumamoto; Tatsuhiko Ooie, National Hospital Organization Oita Medical Center, Oita, Oita; Masahiro Yoshinaga, Beppu Medical Center, Beppu, Oita; Masahito Aburaya, Koga General Hospital, Miyazaki, Miyazaki; Yoshihide Ushitani, Clinic Ushitani, Miyazaki, Miyazaki; Masataka Inakura and Shou Nagai, Ebino-Centro Clinic, Ebino, Miyazaki.

\section{References}

1. Nakashima S, Arai S, Mizuno Y, Yoshino K, Ando S, Nakamura $\mathrm{Y}$, et al. A clinical study of Japanese patients with ulcer induced by low-dose aspirin and other non-steroidal anti-inflammatory drugs. Aliment Pharmacol Ther. 2005;21(Suppl 2):60-6.

2. Nema H, Kato M, Katsurada T, Nozaki Y, Yotsukura A, Yoshida $\mathrm{I}$, et al. Investigation of gastric and duodenal mucosal defects caused by low-dose aspirin in patients with ischemic heart disease. J Clin Gastroenterol. 2009;43:130-2.

3. Nakayama M, Iwakiri R, Hara M, Otani H, Shimoda R, Tsunada $\mathrm{S}$, et al. Low-dose aspirin is a prominent cause of bleeding ulcers in patients who underwent emergency endoscopy. J Gastroenterol. 2009;44:912-8.

4. Shiotani A, Sakakibara T, Yamanaka Y, Imamura H, Tarumi K, Manabe N, et al. Upper gastrointestinal ulcer in Japanese patients taking low-dose aspirin. J Gastroenterol. 2009;44:126-31.

5. Bhatt DL, Scheiman J, Abraham NS, Antman EM, Chan FK, Furberg CD, et al. ACCF/ACG/AHA 2008 expert consensus document on reducing the gastrointestinal risks of antiplatelet therapy and NSAID use. Am J Gastroenterol. 2008;103: 2890-907.

6. Sung JJ, Lau JY, Ching JY, Wu JC, Lee YT, Chiu PW, et al. Continuation of low-dose aspirin therapy in peptic ulcer bleeding, a randomized trial. Ann Intern Med. 2010;152:1-9.

7. Lai KC, Lam SK, Chu KM, Wong BC, Hui WM, Hu WH, et al. Lansoprazole for the prevention of recurrence of ulcer complications from long-term low-dose aspirin use. N Engl J Med. 2002;346:2033-8.

8. Yeomans N, Lanas A, Labenz J, van Zanten SV, van Rensburg C, Rácz I, et al. Efficacy of esomeprazole (20 mg once daily) for reducing the risk of gastroduodenal ulcers associated with continuous use of low-dose aspirin. Am $\mathbf{J}$ Gastroenterol. 2008;103:2465-73.

9. Taha AS, McCloskey C, Prasad R, Bezlyak V. Famotidine for the prevention of peptic ulcers and oesophagitis in patients taking low-dose aspirin (FAMOUS): a phase III, randomised, doubleblind, placebo-controlled trial. Lancet. 2009;374:119-25.

10. Ng FH, Wong SY, Lam KF, Chu WM, Chan P, Ling YH, et al. Famotidine is inferior to pantoprazole in preventing recurrence of aspirin-related peptic ulcers or erosions. Gastroenterology. 2010;138:82-8.

11. Ishimori A, Yamagata S. Therapeutic effect of pirenzepine dihydrochloride on gastric ulcer evaluated by a double-blind 
controlled clinical study. Arzneimittelforschung. 1982;32:55665 .

12. Lyon DT. Efficacy and safety of famotidine in the management of benign gastric ulcers. Am J Med. 1986;81:33-41.

13. Lanza FL, Royer GL, Nelson RS, Chen TT, Seckman CE, Rack MF. A comparative endoscopic evaluation of the damaging effects of nonsteroidal anti-inflammatory agents on the gastric and duodenal mucosa. Am J Gastroenterol. 1981;75:17-21.

14. Asaka M, Kato M, Takahashi S, Fukuda Y, Sugiyama T, Ota H, et al. Guidelines for the management of Helicobacter pylori infection in Japan: 2009 revised edition. Helicobacter. 2010;15:1-20.

15. Chan FKL, Chung S, Suen BY, Lee YT, Leung WK, Leung VKS, et al. Preventing recurrent upper gastrointestinal bleeding in patients with Helicobacter pylori infection who are taking lowdose aspirin or naproxen. N Engl J Med. 2001;344:967-73.
16. Fukase K, Kato M, Kikuchi S, Inoue K, Uemura N, Okamoto S, et al. Effect of eradication of Helicobacter pylori on incidence of metachronous gastric carcinoma after endoscopic resection of early gastric cancer: an open-label, randomised controlled trial. Lancet. 2008;372:392-7.

17. Iijima K, Ohara S, Sekine H, Koike T, Kato K, Asaki S, et al. Changes in gastric acid secretion assayed by endoscopic gastrin test before and after Helicobacter pylori eradication. Gut. 2000;46:20-6.

18. Maeda S, Ogura K, Yoshida H, Kanai F, Ikenoue T, Kato N, et al. Major virulence factors, VacA and CagA, are commonly positive in Helicobacter pylori isolates in Japan. Gut. 1998;42:338-43.

19. Nakajima S, Nishiyama Y, Yamaoka M, Yasuoka T, Cho E. Changes in the prevalence of Helicobacter pylori infection and gastrointestinal diseases in the past 17 years. J Gastroenterol Hepatol. 2010;25(Suppl 1):S99-110. 